\title{
The effect of intra-molecular relaxations on the damping of longitudinal and transverse phonons in polysiloxanes studied by Brillouin spectroscopy
}

\author{
H. Kriegs and G. Meier \\ Institut für Festkörperforschung, Forschungszentrum Jülich, 52425 Jülich, Germany \\ J. Gapinski and A. Patkowski \\ Institute of Physics, A. Mickiewicz University, Umultowska 85, 61-614, Poznan, Poland
}

\begin{abstract}
The effect of intra-molecular relaxations on the damping of longitudinal and transverse phonons was studied in poly(methylphenylsiloxane) (PMPS) and poly(ethylmethylsiloxane) (PEMS) polymers by means of Brillouin spectroscopy. It is shown that studies of the polarized and depolarized Brillouin spectra as functions of temperature and pressure allow for the separation of the contributions of the internal and structural relaxations to the damping of longitudinal and transverse phonons, respectively. In polymers with intra-molecular relaxations these processes contribute not only to the damping of longitudinal phonons, according to theoretical predictions, but also transverse phonons, in contradiction to the theory.
\end{abstract}




\section{Introduction}

In liquids, propagating density fluctuations exist in the form of longitudinal and transverse phonons. The velocity and damping of these phonons at high frequencies of the order of a few $\mathrm{GHz}$ can be studied by means of the polarized (VV - longitudinal phonons) and depolarized (VH - transverse phonons) Brillouin spectroscopy. The VV and VH Rayleigh-Brillouin spectrum consists of the central Rayleigh line and two Brillouin lines which are symmetrically shifted by $\pm \omega_{\mathrm{B}}$ and have the half-width at half heights $(\mathrm{HWHH}) \Gamma_{\mathrm{B}}$. The Brillouin shift $\omega_{\mathrm{B}}$ is the measure of the sound velocity v: $\omega_{\mathrm{B}}=\mathrm{qv}$, where $\mathrm{q}=(4 \pi \mathrm{n} \sin \theta / 2) / \lambda$ is the length of the scattering vector, $\mathrm{n}$ is the refractive index of the scattering medium, $\theta$ - the scattering angle and $\lambda$ - the wavelength of the incident light in vacuum. The phonons are damped due to the relaxation processes present in the liquid and the measure of this damping is the Brillouin line width $\Gamma_{\mathrm{B}}$. In the case of a single relaxation process of a relaxation time $\tau_{\mathrm{s}}$ the classical acoustic relaxation theory predicts the maximum of damping when $\omega_{\mathrm{B}} \tau_{\mathrm{s}}=1$. In the case of supercooled liquids the situation is much more complicated due to the presence of several relaxation processes. Additional complications might result from the fact that intramolecular relaxation processes in the liquid might also contribute to the damping of phonons.

Theories describing the propagation and damping of longitudinal and transverse phonons in terms of the structural relaxation, i.e. translational motion of the molecules and translational-rotational coupling, have been developed, improved and tested experimentally [1,2 and references cited therein]. Usually they did not take into account the coupling of intra-molecular relaxations to the structural relaxation. 
For liquids which consist of molecules having internal relaxation, a theoretical model was developed in the limit of weak coupling of intra-molecular relaxations to the translational degrees of freedom [3]. It was shown that internal processes contribute to the damping of longitudinal but not transverse phonons.

In order to estimate the contribution of internal relaxations to the broadening of the Brillouin lines (damping of phonons) it is necessary to separate this contribution from that of structural relaxation. This can be achieved by studying the pressure dependence of $\Gamma_{B}[4,5]$, since the pressure dependence of the structural relaxation times $\operatorname{dlog} \tau_{\S} / \mathrm{dP} \sim 5 \times 10^{-3}$ $\operatorname{bar}^{-1}[6]$ is much stronger than that of the internal relaxation time $\operatorname{dlog} \tau_{\text {int }} / \mathrm{dP} \sim 2-5 \times 10^{-4}$ $\operatorname{bar}^{-1}[7,8,9]$. The efficiency of the phonon damping depends mainly on the separation in frequency between the relaxation frequency $1 / \tau$ and the phonon frequency $\omega_{\mathrm{B}}$. Thus the contribution of the structural relaxation to the $\Gamma_{\mathrm{B}}$ should change from zero at the glass transition temperature $T_{\mathrm{g}}$ to a maximum, when $\omega_{\mathrm{B}} \tau_{\mathrm{s}}=1$, while the internal relaxations should contribute almost equally at all temperatures, since their relaxation frequency is changing very little with temperature and pressure.

To the best of our knowledge, no systematic study of the effect of intramolecular relaxations in polymeric supercooled liquids on the damping of phonons has been performed.

Li [10] have studied the phonon propagation and damping in poly(alkylmethacrylates), where alkyl stands for methyl, ethyl and butyl-side chains. It was found that the phonon line-widths $\Gamma_{\mathrm{B}}$ adopt a rather high residual value of about $500 \mathrm{MHz}(\mathrm{HWHH})$ at high temperatures. The form of the dispersion curve $\Gamma_{B}(T)$ flattens the longer the side chain length is. This trend is even stronger when a hexyl side-chain poly(methacrylate) is 
studied [11]. Moreover, the maximum of dispersion is shifted towards lower $\mathrm{T}$ with increasing side chain length. Patterson [12] has pointed out that the reason for the maximum of phonon dispersion lies in the dispersion of secondary relaxations, since in a typical transition map at the hypersonic frequencies we find the extrapolated (to high temperatures) relaxation times of the secondary processes measured at low temperatures. This phenomenon in long side chain poly(alkylmethacrylate)s is discussed in terms of the dispersion of polyethylene-like nano-domains [13]. In a Brillouin study on poly(methylphenylsiloxane) PMPS also a large residual line-width of the order of 240 $\mathrm{MHz}$ at high temperatures was found [14]. This could be assigned to the relaxation of the phenyl group as was shown in a quasielastic neutron scattering study [15]. Thus, the excess line width obviously can be ascribed to some intra-molecular relaxation processes, however, distinguishing from the potential influence of the inter-molecular processes is difficult, because that largely depends on structural details of the molecules and moreover we do not have a molecular model to describe the form of the dispersion of the phonon damping. The simplest approach to that was a single relaxation model, despite it was already criticized by Patterson [12]. He claimed that the distribution of relaxation times is the reason for the relatively large residual Brillouin line-width. Later Fytas [16] showed in a study of siloxane oligomers that at least in the vicinity of maximum loss the single relaxation model is a good approximation. As we have shown previously [4,5], the most reliable way to separate the contributions of internal and structural relaxations to the broadening of the Brillouin lines and the damping of phonons, is to measure the pressure dependence of the Brillouin spectra. 
In some cases an assignment to structural (inter-) and intra-molecular relaxations can be performed on a molecular level, as it has been done in the case of two chemically very similar (Fig.1) polymeric supercooled liquids: PMPS and PEMS $[17,18,6]$. The only difference in their chemical structure is the presence of a phenyl ring in PMPS which replaces the ethyl group in PEMS. In PMPS in addition to the structural relaxation an intramolecular process is present which is assigned to the phenyl flip. In PEMS this internal process is not present. This well identified difference in the internal dynamics makes this pair of polymeric liquids a perfect choice to study the effects of internal relaxations on damping of phonons.

Recently $[4,5]$ we have shown, that in the case of molecules with weak internal relaxations (BMMPC, OTP) only the damping of the longitudinal phonons is affected, in agreement with the theoretical predictions for the weak coupling limit [3]. Strong internal relaxations (BMPC) contribute to damping of both longitudinal and transverse phonons. No theoretical model is available to explain this effect. The strongest arguments in our studies were provided by the idea of separating the contribution of structural and internal relaxations to the broadening of the Brillouin lines by studying the pressure dependence of the Brillouin spectra.

In this work we present systematic studies of propagation and damping of longitudinal and transverse phonons in PMPS and PEMS in a broad temperature and pressure range. The results obtained for these two liquids are compared with the corresponding data obtained for low molecular weight liquids. We show that the contribution of internal relaxations to the broadening of the Brillouin spectra can be estimated at any temperature from the pressure dependent studies. By comparing the data obtained for a liquid with 
well identified strong internal relaxations (PMPS) with those for a liquid with weak internal processes we show the role of intramolecular processes in the damping of longitudinal phonons.

\section{Theoretical background}

The isotropic Brillouin spectrum can be calculated from the experimental polarized (VV) and depolarized (VH) Brillouin spectra:

$$
I_{I s o}(q, \omega)=I_{V V}(q, \omega)-\frac{1}{d} I_{V H}(q, \omega)
$$

where $d$ is the depolarization ratio. The isotropic spectrum decays to zero at frequencies beyond the Brillouin peak $(\omega>40 \mathrm{GHz})$. Thus, the depolarization ratio can be obtained from these high frequency parts of the VV and VH Brillouin spectra.

The experimental isotropic Brillouin spectrum can be expressed as a convolution of the dynamic structure factor $\mathrm{S}(\mathrm{q}, \omega)$ (describing the spectrum of density fluctuations in the system) and the instrumental resolution function $R(\omega)$ :

$$
I_{\text {Iso }}(q, \omega)-I_{0} \int R\left(\omega^{\prime}\right) S\left(q, \omega-\omega^{\prime}\right) d \omega^{\prime}
$$

where $\mathrm{I}_{0}$ is a constant.

The dynamic structure factor can be expressed in terms of the memory function $\mathrm{m}(\mathrm{q}, \omega)$ using the simple generalized hydrodynamic formalism $[19,20]$ : 


$$
S(q, \omega)=\frac{2 v_{0}^{2} q^{2}}{\omega} \operatorname{Im}\left[\omega^{2}-\omega_{T}^{2}(q)-i \omega m_{q}(\omega)\right]^{-1},
$$

where $\mathrm{v}_{0}=\mathrm{k}_{\mathrm{B}} \mathrm{T} / \mathrm{M}, \quad(\mathrm{M}$ is the molecular mass of the scattering molecule), $\left.\omega_{\mathrm{T}}^{2}(\mathrm{q} \rightarrow 0)\right)=\mathrm{q}^{2} \mathrm{c}_{0}^{2} / \gamma, \mathrm{c}_{0}$ is the adiabatic sound velocity and $\gamma=\mathrm{c}_{\mathrm{P}} / \mathrm{c}_{\mathrm{V}}$ is the ratio of specific heats at constant pressure and volume, respectively.

In the case of supercooled liquids the memory function, in addition to the usually negligible contribution of the thermal diffusivity term, contains terms related to three relaxation processes: (i) the structural relaxation contribution in the form of the ColeDavidson process, (ii) fast relaxation in the form of the Debye process, and (iii) a frequency independent microscopic or instantaneous process:

$$
K(\omega)=\frac{q^{2} \Delta_{\alpha}^{2}}{i \omega \rho}\left[1-\frac{1}{\left(1+i \omega \tau_{\alpha}\right)^{\beta_{C D}}}\right]+\frac{q^{2} \Delta_{f}^{2} \tau_{f}}{\rho\left(1+i \omega \tau_{f}\right)}+\gamma_{0} q^{2}
$$

where $\rho$ is the liquid density, $\Delta_{\alpha}, \Delta_{\mathrm{f}}$ and $\tau_{\alpha}, \tau_{\mathrm{f}}$ are the amplitudes and relaxation times of the structural $(\alpha)$ and fast (f) relaxations, respectively, $\beta_{\mathrm{CD}}$ is the Cole-Davidson exponent and $\gamma_{0}$ is the amplitude of the microscopic process. Using this phenomenological approach it was possible to fit the shape of the isotropic Brillouin spectra of some glass forming liquids [19]. In this kind of analysis all relaxation processes are simply added up in the memory function without taking into account their physical origin, coupling between them and their coupling to the physical parameters being measured in a given experiment. 
A theory describing explicitly the contribution of internal relaxations to the damping (broadening) of phonons (Brillouin lines) was developed by Zwanzig [3] in the limit of weak coupling of internal processes to the centre-of-mass motions. In this limit it is assumed that the contribution due to coupling of internal and centre-of mass motions to the total Hamiltonian (consisting of parts related to the centre-of-mass and internal motions) is very small [3]. The consequences of this limit are that, to a good approximation, the averages and time derivatives of functions depending on translational and internal variables can be calculated independently.

The longitudinal phonon linewidth is given in the classical hydrodynamic theory by [21]:

$$
\Gamma_{B}=\frac{q^{2}}{2 m \rho}\left(\eta_{v}+\frac{4}{3} \eta\right)
$$

after neglecting the contribution due to the thermal conductivity term. Here $m$ is the mass of the molecule, $\rho$ is the number density of molecules in the liquid and $\eta$ and $\eta_{v}$ are the shear and bulk viscosities, respectively.

In the weak coupling limit, the bulk viscosity, determining the width of the Brillouin line, is given by [3]:

$$
\eta_{v}(\omega)=\eta_{v C}(0)+\frac{\left(c_{P}-c_{V}\right) c_{I}}{\left(c_{V}-c_{I}\right) c_{P} \chi_{S}} \int_{0}^{\infty} d t \exp (-i \omega t) \Phi(t)
$$

where $\eta_{v C}(0)$ is the translational contribution to the bulk viscosity, $c_{V}, c_{P}$, and $c_{I}$ are the specific heats at constant volume and pressure and of the internal modes, $\chi_{s}$ is the 
adiabatic compressibility and $\Phi(t)$ is the correlation function of the fluctuations of the internal energy. The second term of Eq.6 represents the additional contribution of the internal process to the bulk viscosity and thus to the width of the polarized Brillouin line. If the $\Phi(t)$ function is exponential:

$$
\Phi(t)=\exp (-t / \tau)
$$

then this additional contribution is given by:

$$
\Delta \eta_{v}(\omega)=\frac{\left(c_{P}-c_{V}\right) c_{I}}{\left(c_{V}-c_{I}\right) c_{P} \chi_{S}} \frac{\tau}{1+i \omega \tau}
$$

This theory predicts no contribution of internal relaxations to the width of the depolarized Brillouin line and the damping of the transverse phonons.

\section{Materials and methods}

\section{Materials}

The chemical structure of the PMPS and PEMS polymers is shown in Fig.1. Both polymers were synthesized at the Max Planck Institute for Polymer Research, Mainz by Thomas Wagner. The weight and number average molecular weights of both polymers amounted to: $\mathrm{M}_{\mathrm{w}}(\mathrm{PMPS})=10500 \mathrm{Da}, \mathrm{M}_{\mathrm{w}}(\mathrm{PEMS})=14860 \mathrm{Da}, \mathrm{M}_{\mathrm{N}}(\mathrm{PMPS})=10100 \mathrm{Da}$, $\mathrm{M}_{\mathrm{N}}(\mathrm{PEMS})=13400$ Da, and the polydispersities $\mathrm{M}_{\mathrm{w}} / \mathrm{M}_{\mathrm{n}}(\mathrm{PMPS})=1.04$, and $\mathrm{M}_{\mathrm{w}} / \mathrm{M}_{\mathrm{n}}$ $(\mathrm{PEMS})=1.11$, rescpectively. The corresponding glass transition temperatures measured by means of the DSC amounted to $\mathrm{T}_{\mathrm{g}}(\mathrm{PMPS})=236 \mathrm{~K} \quad$ and $\mathrm{T}_{\mathrm{g}}(\mathrm{PEMS})=138 \mathrm{~K}$. 
The samples were annealed under vacuum at high temperature above $T_{g}$ and then were filtered into the light scattering cells using a Millipore filter (pore size $0.45 \mu \mathrm{m}$ ).
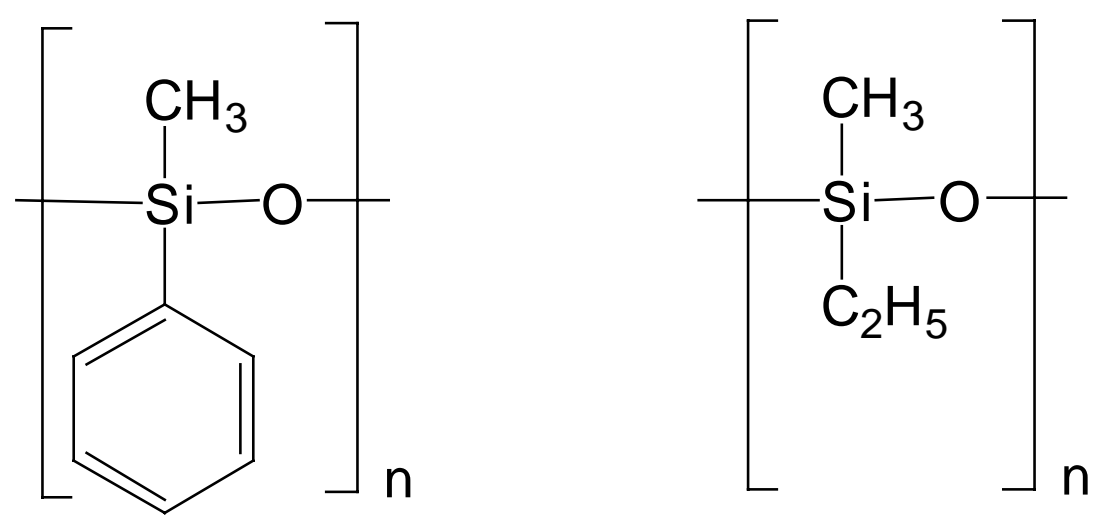

Fig. 1. Structure of PMPS (left) and PEMS (right)

\section{Experimental setup}

Polarized (VV) and depolarized Rayleigh-Brillouin spectra were measured in the backscattering and $90^{\circ}$ geometry, respectively, using a 6-pass Tandem Fabry-Perot interferometer (JRS Instruments, Switzerland). A Nd:YAG laser (Coherent DPSS 532200) operating at $\lambda=532 \mathrm{~nm}$ at a power of $200 \mathrm{~mW}$ and Ar-ion laser (Spectra Physics, Model2060) at $\lambda=514.5 \mathrm{~nm}$ and a power not exceeding $200 \mathrm{~mW}$ were used as incident light sources. Pressure dependent studies were performed in a home-made high pressure cell operating in the pressure range of 1-2000 bar. The experimental setup is described in detail elsewhere [22].

\section{Data analysis}

Since no theoretical form of the entire Rayleigh - Brillouin spectrum is available for liquids with structural and internal relaxations, we used a simple approach in the analysis 
of our data in order to obtain the position $\omega_{\mathbf{B}}$ and the width of $\Gamma_{\mathbf{B}}$ of the Brillouin lines. Basically, the experimental spectra were fitted using a model function, consisting of the central Rayleigh peak and two symmetrically positioned Brillouin peaks according to eq.9. This model function was convoluted with the experimental instrumental function which was determined from a reference beam measurement. In the case of a $\mathrm{VH}$ spectrum for the central peak a sum of a Cole-Davidson function ( $\alpha$-relaxation) plus an algebraic high frequency term with $g$ as an amplitude [23] was used. For the VV-spectra with the central part mainly cut, one Lorentzian was found to be sufficient to represent the fast process and to fit the central line including the high frequency wings. The Brillouin peaks were described by Lorentzians (last two terms in eq.9). The normal Lorentzian form was modified according to Berne and Pecora [21] in order to account for non-Lorentzian corrections and rendering the Brillouin lines asymmetric. Thus, the model function has the form:

$$
\begin{aligned}
I(\omega)= & I_{d a r k}+\frac{I_{\alpha}}{\omega} \operatorname{Im}\left[1-\left(\frac{1}{1+i \omega \tau_{s}}\right)^{\beta_{C D}}+i g \omega^{0.33}\right] \\
& +I_{f} \frac{\Gamma_{f}}{\omega^{2}+\Gamma_{f}^{2}}+I_{B} \cdot\left[\frac{\Gamma_{B}-s\left(\omega-\omega_{B}\right)}{\left(\omega-\omega_{B}\right)^{2}+\Gamma_{B}^{2}}+\frac{\Gamma_{B}+s\left(\omega+\omega_{B}\right)}{\left(\omega+\omega_{B}\right)^{2}+\Gamma_{B}^{2}}\right]
\end{aligned}
$$

Here $I_{d a r k}$ denotes the baseline of the measured spectrum, $I_{\alpha}, I_{f}$ and $I_{B}$ are the intensities of the $\alpha$-relaxation, the fast process and Brillouin lines, respectively, $\Gamma_{f}$ is the width of the fast process, $\omega_{\mathrm{B}}$ and $\Gamma_{B}$ are the position and width of the Brillouin lines, respectively. Parameter $s$ determines the asymmetry of the Brillouin peaks. From a fit of eq. 9 to our experimental data the Brillouin shift $\omega_{\mathrm{B}}$ and Brillouin line width $\Gamma_{\mathrm{B}}$ (the half width at half heights) could be obtained. An example of this data analysis is given in Fig.2. 


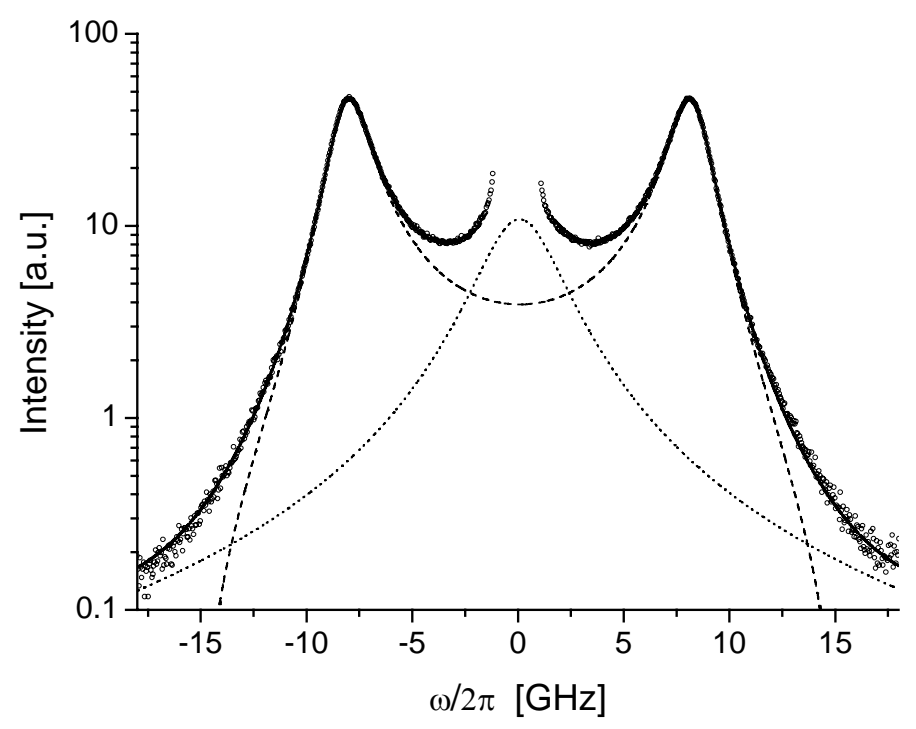

Fig. 2. The polarized (VV) Brillouin spectrum of PEMS measured at ambient pressure and temperature $-23^{\circ} \mathrm{C}$. Full line is the fit of eq.9 to the data. Dashed lines show respective contributions to the full expression according to eq.9.

Any kind of more sophisticated analysis would likely give access to more parameters, but since this paper is only concerned with interpretations on the level of shift and line width we have chosen this rather simple approach.

\section{Results and discussion}

\section{IV.1. Results}

IV.1.1. Comparison of the VV Brillouin spectra of PMPS and PEMS at ambient pressure

Polarized (VV) Brillouin spectra were measured at ambient pressure for PMPS and PEMS in the back-scattering geometry for $\lambda=532 \mathrm{~nm}$ in a broad temperature range from 
$T_{g}$ to about $T_{g}+200 \mathrm{~K}$. The experimental spectra for PEMS are shown in Fig.3. The corresponding spectra for PMPS are shown in Fig. S1 in the supplement [24].

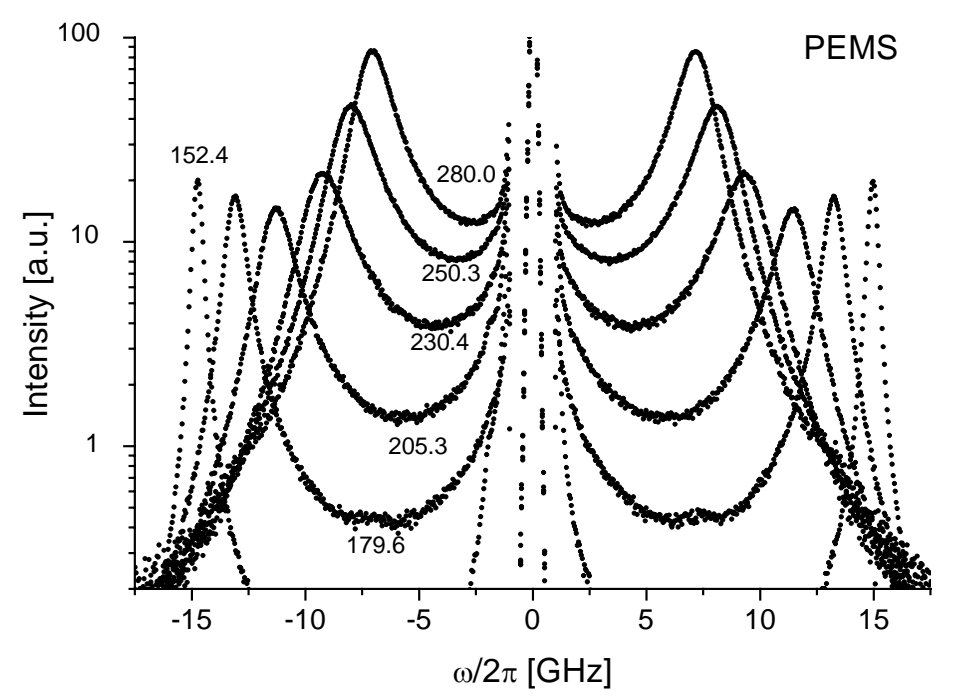

Fig. 3. Polarized (VV) Brillouin spectra measured at ambient pressure for PEMS in the back-scattering geometry for $\lambda=532 \mathrm{~nm}$ at temperatures, in [K] indicated in the figure.

The central line is removed from the spectra because in this geometry it is dominated by the stray light reflected from the sample. Good quality of the data allows for an exact determination of the position $\omega_{\mathrm{B}}$ and the half width at half heights $\Gamma_{\mathrm{B}}$ of the Brillouin peaks. These $\Gamma_{\mathrm{B}}$ values, recalculated for the scattering angle of $90^{\circ}$ and $\lambda=514.5 \mathrm{~nm}$, are plotted for PMPS and PEMS versus T- $\mathrm{T}_{\mathrm{g}}$ in Fig. 4. The corresponding $\omega_{\mathrm{B}}$ values are plotted in Fig. S2 in the supplement [24]. The temperature dependence of $\omega_{\mathrm{B}}$ is similar for both polymers. However, there are two important differences in the temperature dependence of $\Gamma_{\mathrm{B}}$ : (i) the maximum of the dependence $\Gamma_{\mathrm{B}}\left(T-T_{\mathrm{g}}\right)$ for PMPS is much broader than that for PEMS, and (ii) the residual width at the glass transition temperature $\Gamma_{\mathrm{B}}\left(T_{\mathrm{g}}\right)$ is also larger for PMPS than for PEMS. 


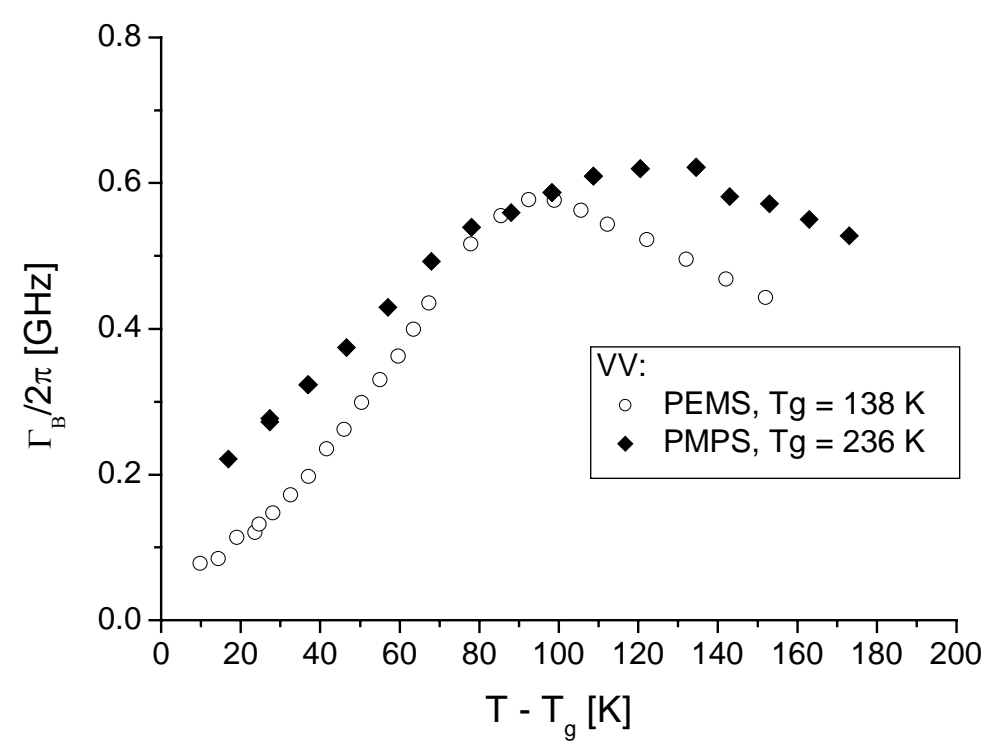

Fig. 4. Temperature dependence of the half width at half heights $\Gamma_{B}$ of the polarized Brillouin peak measured for PMPS and PEMS at ambient pressure, rescaled to $\theta=90^{\circ}, \lambda=514.5 \mathrm{~nm}$.

\section{IV.1.2. Pressure dependence of the polarized Brillouin spectra of PMPS}

The pressure dependence of the polarized Brillouin spectra in the temperature range close to $T_{\mathrm{g}}$ could be measured only for PMPS, because the temperatures close to $\mathrm{T}_{\mathrm{g}}=138 \mathrm{~K}$ of PEMS could not be reached in our high pressure light scattering setup. The quality of the experimental spectra was similar to that shown in Fig. 3. The pressure dependence of the half width $\Gamma_{\mathrm{B}}$ of the longitudinal phonon peaks at temperatures of $-21,0$ and $20^{\circ} \mathrm{C}$ is 
shown in Fig. 5 and a corresponding dependence of the position $\omega_{\mathrm{B}}$ is shown in Fig.S3 in the supplement [24].

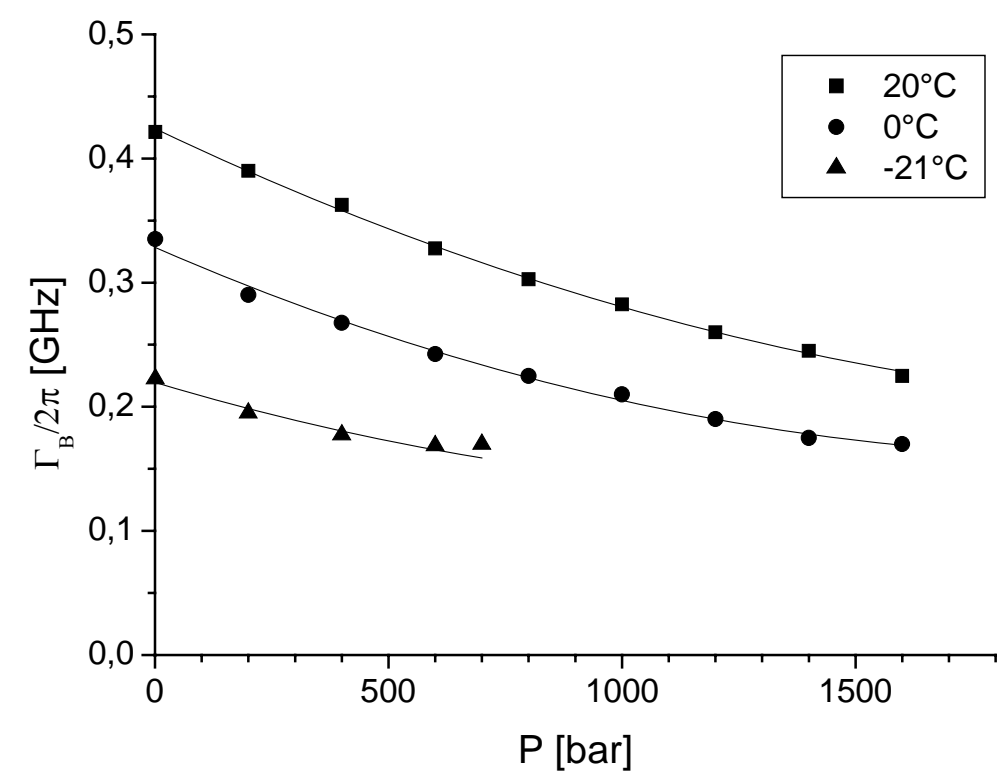

Fig. 5. Pressure dependence of the half width of the polarized Brillouin peak measured for PMPS at indicated temperatures. The solid lines represent the fit using Eq.10.

As already observed for low molecular weight liquids [5], the Brillouin width $\Gamma_{\mathrm{B}}$ is decreasing with increasing pressure, reaching a high pressure plateau value. In order to obtain this value $\Gamma_{0}$, the data was analyzed using the formula:

$$
\Gamma_{B}(P)=\Gamma_{0}(T)+A(T) \exp \left(\frac{-D_{P} P}{P_{0}-P}\right)
$$

where $\Gamma_{0}(\mathrm{~T})$ is the residual width at $\mathrm{T}_{\mathrm{g}}$, due to internal relaxations, $\mathrm{A}(\mathrm{T})$ is the amplitude, $\mathrm{D}_{\mathrm{P}}$ is a fit parameter identical for all temperatures and $\mathrm{P}_{0}$ is the parameter characterizing 
the pressure dependence of the structural ( $\alpha$-) relaxation obtained from our earlier high pressure photon correlation spectroscopy study [6]. The temperature dependence of $\Gamma_{0}$ and A is shown in Fig.6.

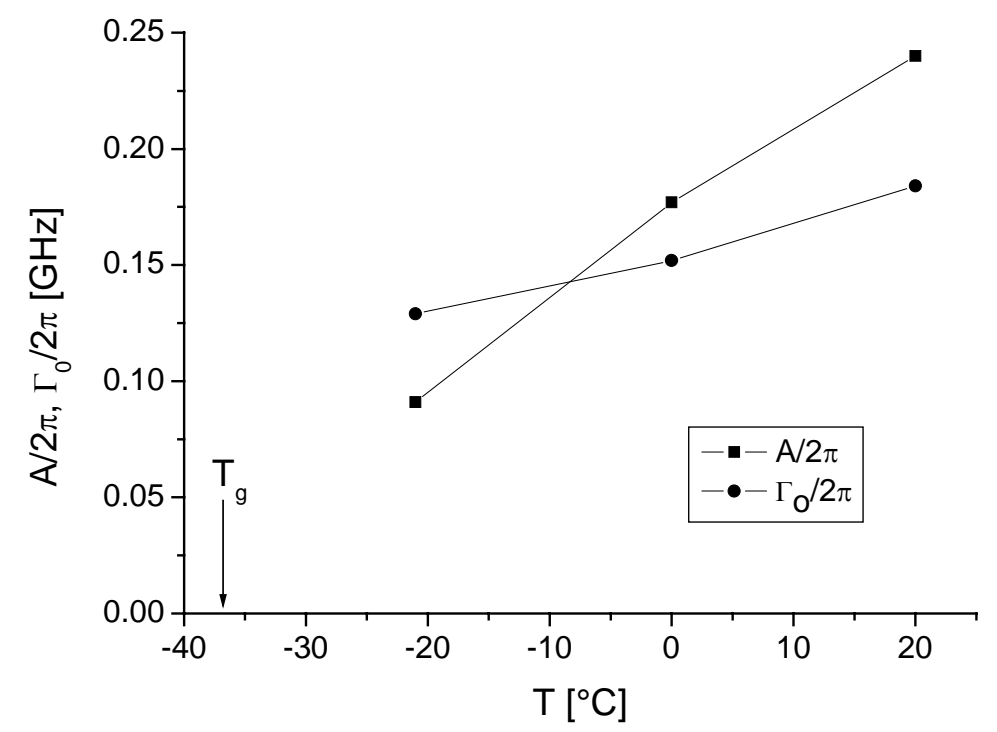

Fig. 6. Temperature dependence of $\Gamma_{0}$ and A obtained for PMPS from the polarized

\section{Brillouin spectra.}

\section{IV.1.3. Pressure dependence of the depolarized (VH) Brillouin spectra of PMPS}

The temperature and pressure dependence of the depolarized Brillouin spectra, which are due to transverse phonons, was also extensively studied for PMPS in the $90^{\circ}$ scattering geometry. Typical sets of temperature dependent spectra measured at a pressure of 600 bar are shown in Fig. 7. Pressure dependent spectra measured at a temperature of $12^{\circ} \mathrm{C}$ Fig.S4 can be seen in the supplement [24]. 


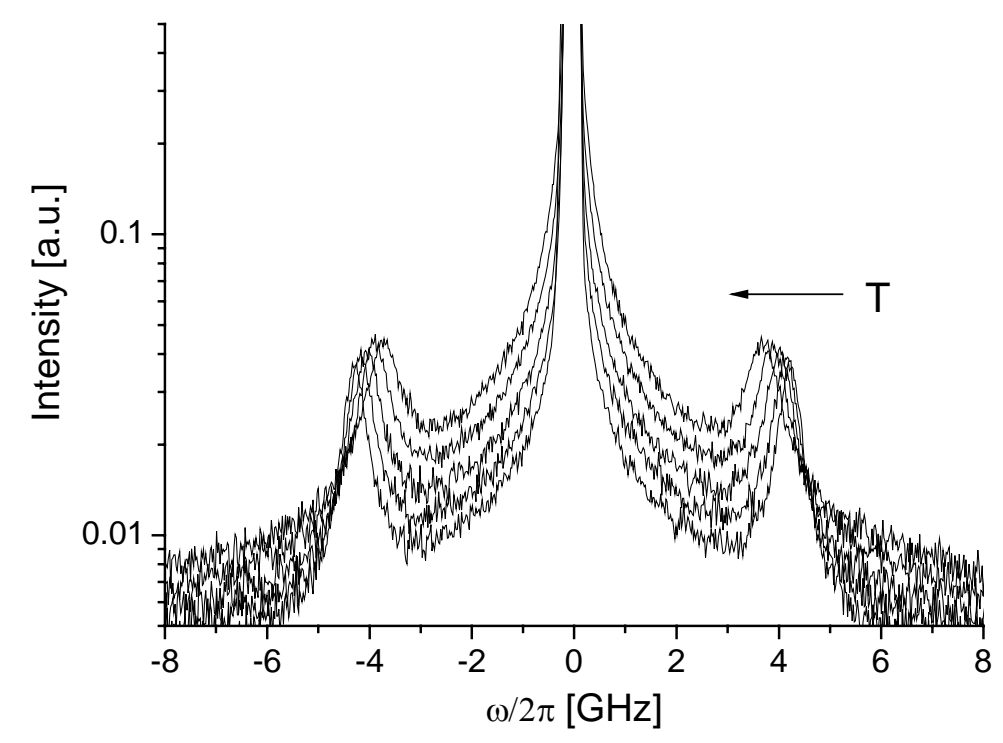

Fig. 7. Depolarized (VH) Brillouin spectra measured for PMPS at a constant pressure of 600 bar and temperatures of $4.3,8.1,12,17.6$ and $24.3^{\circ} \mathrm{C}$.

As one can see in these figures, the transverse Brillouin peak in the depolarized spectra is moving to lower frequencies and getting broader with increasing temperature or decreasing pressure.

In order to separate the relaxational contribution to the Rayleigh-Brillouin spectra from the Brillouin peaks, the depolarized spectra were also measured in the back-scattering $\left(180^{\circ}\right)$ geometry. In this geometry only the relaxational part of the spectrum is visible, while the transverse Brillouin peaks disappear. A superposition of the depolarized Brillouin spectra measured in the $90^{\circ}$ and $180^{\circ}$ scattering geometries for PMPS at a pressure of 200 bar and temperatures of 281 and $291 \mathrm{~K}$ is shown in Fig.8. 


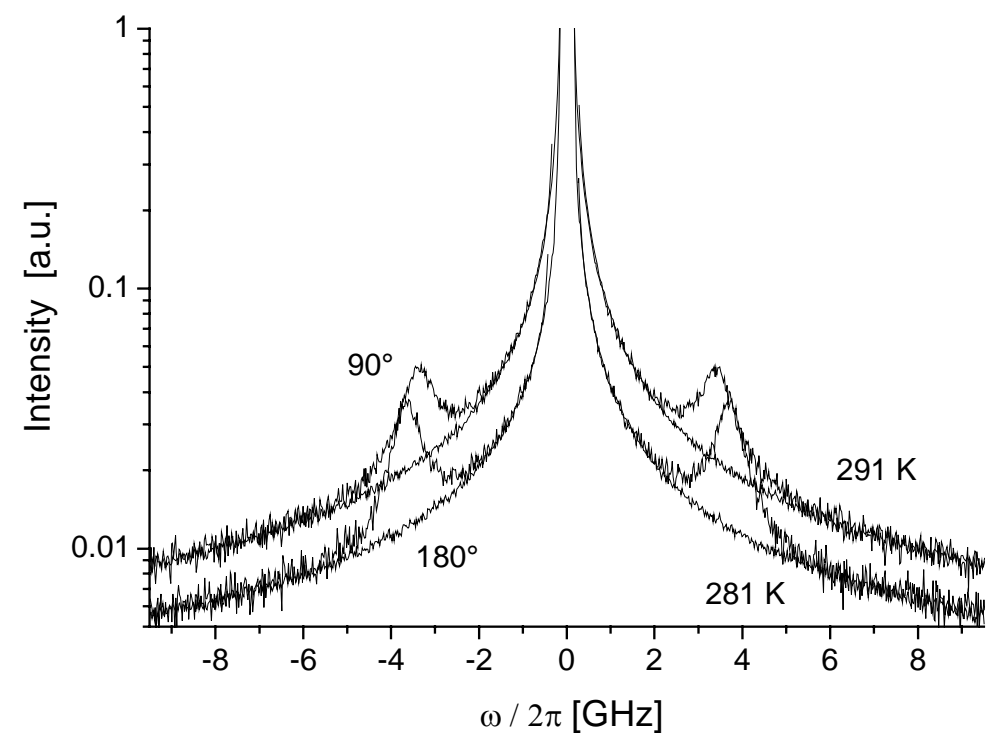

Fig. 8. Comparison of the depolarized Brillouin spectra measured for PMPS in the $90^{\circ}$ and $180^{\circ}$ scattering geometries at a pressure of 200 bar and temperatures of 281 and $291 \mathrm{~K}$.

In Fig. 8 it is clearly evident that the central Rayleigh peak, including the high frequency wings, is identical in both $90^{\circ}$ and $180^{\circ}$ spectra and allows an exact determination of the relaxational contribution. Thus, the Brillouin contribution can be obtained as a difference between the $90^{\circ}$ and $180^{\circ}$ spectra.

IV.1.4. Temperature and pressure dependence of $\omega_{\mathrm{BT}}(\mathrm{P}, \mathrm{T}) \Gamma_{\mathrm{BT}}(\mathrm{P}, \mathrm{T}), \Gamma_{0}(\mathrm{P}, \mathrm{T})$,

From the analysis of the experimental data using Eq.9 and the values of the structural relaxation times $\tau_{\mathrm{S}}$ and $\beta_{\mathrm{CD}}$ obtained earlier [6] as well as the width of the fast process $\Gamma_{\mathrm{f}}$ taken from literature [15] we can obtain the position $\omega_{\mathrm{BT}}$ and width $\Gamma_{\mathrm{BT}}$ of the transverse Brillouin peaks as a function of temperature and pressure. The pressure dependence of 
$\Gamma_{\mathrm{BT}}$ obtained for PMPS at several temperatures is shown in Fig.9. The pressure dependence of the corresponding $\omega_{\mathrm{B}}$ is shown in Fig.S5 in the supplement [24]. The solid lines in Fig.9 represent the result of the fit using Eq.10.

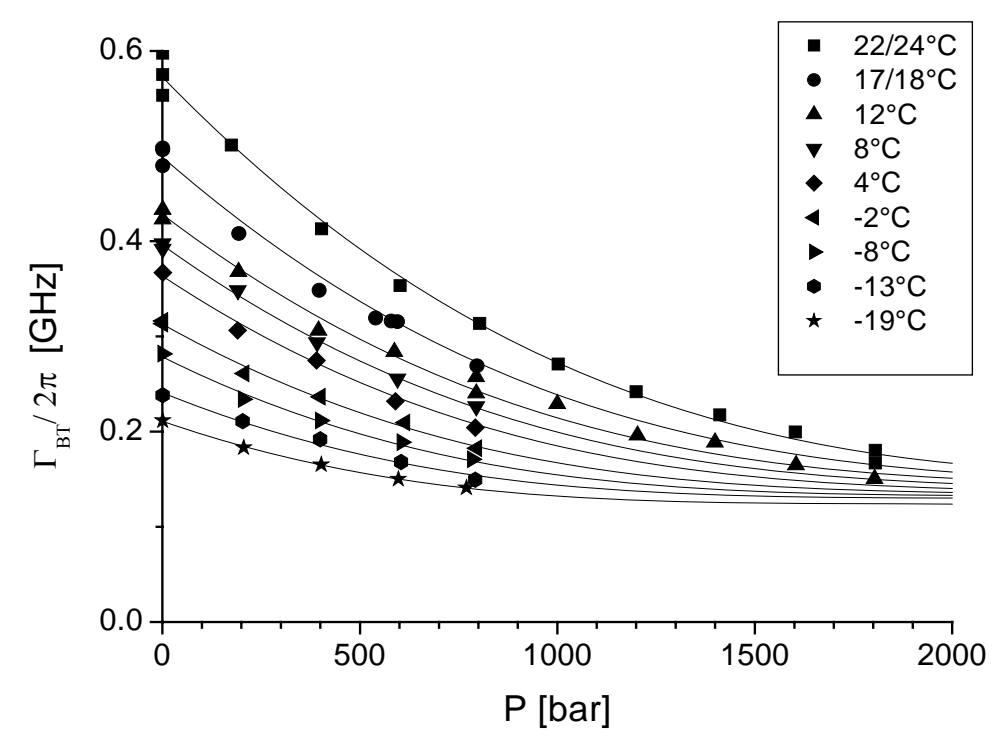

Fig. 9. Pressure dependence of the width of the transverse phonon peak measured for PMPS at temperatures of $-19,-13,-8,-2,4,8,12,18$ and $22^{\circ} \mathrm{C}$ (from bottom to top). The solid lines represent the fit of Eq.10 to the data with a constant $D_{P}=5.6$ and $P_{0}$ taken for each temperature from our previous PCS studies [6].

The pressure dependence of the width of the transverse phonon peaks in PMPS, Fig.9, can be analyzed using Eq.10. On the basis of this analysis the contributions of the internal and structural relaxations to the Brillouin line width and the damping of transverse phonons can be separated and the internal contribution $\Gamma_{0}$ as well as the amplitude of the structural contribution A can be obtained at all temperatures measured. The temperature dependence of $\Gamma_{0}$ and A is shown in Fig.10. 


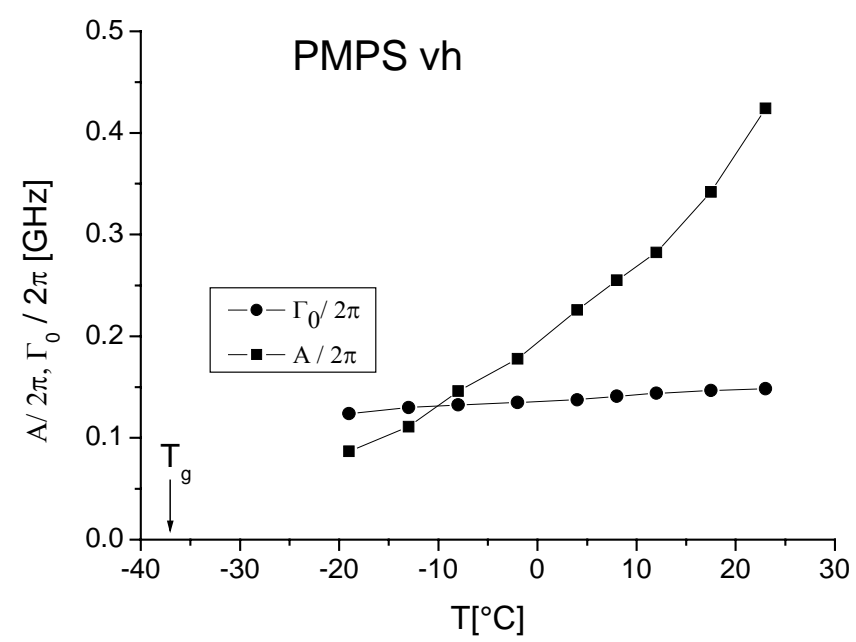

Fig. 10. Temperature dependence of $\Gamma_{0}$ and A obtained for PMPS from the depolarized Brillouin spectra.

As one can see in Fig.10, the contribution of the internal relaxations to the damping of transverse phonons, described by $\Gamma_{0}$, is slightly increasing while the amplitude of the contribution of the structural relaxation is strongly increasing with increasing temperature.

\section{IV.2. Discussion}

IV.2.1. Comparison of the longitudinal phonon damping in PMPS and PEMS at ambient pressure

From the comparison of the temperature dependence of the polarized Brillouin spectra measured for PMPS and PEMS at ambient pressure two important features emerge: (i) 
the width of the Brillouin line at $T_{g}, \Gamma_{B}\left(T_{g}\right)$ is not equal to zero for both polymers, and is much larger for PMPS than for PEMS, and (ii) the temperature dependence of the Brillouin width $\Gamma_{\mathrm{B}}\left(\mathrm{T}-\mathrm{T}_{\mathrm{g}}\right)$ exhibits a much broader maximum for PMPS (than for PEMS), which is shifted to higher temperatures.

Previously, the Brillouin spectra were measured as a function of temperature for a few polysiloxanes: poly(methylphenylsiloxane) (PMPS) and a copolymer poly(dimethylphenylmethylsiloxane) [14] as well as poly(diphenylsiloxane) (PDPS), poly(methylphenylsiloxane) (PMPS) and their model analogs [16]. In all these polymers which contain a phenyl group, a residual Brillouin line width at high temperature equal to $0.25 \mathrm{GHz}$ was found and was assigned to the coupling of intramolecular vibrations to translation of the molecules. This is in a good agreement with our results for PMPS. However, for PEMS (no phenyl group) the residual line width $\Gamma_{\mathrm{B}}\left(\mathrm{T}_{\mathrm{g}}\right)$ was more than a factor of 2 lower, Fig.4. This residual line width can be assigned to the internal relaxations, since at $T_{g}$ the structural relaxation is practically frozen and does not contribute to the phonon damping.

Thus, our comparative Brillouin studies of PMPS and PEMS corroborate the previous assignment of the residual broadening of the polarized Brillouin lines (additional damping of longitudinal phonons) to the coupling of the intramolecular relaxations to the translational degrees of freedom. Indeed, according to the theory [3] developed for the case of weak coupling of internal and translational degrees of freedom, the presence of internal relaxations should result in additional broadening (damping) of the VV Brillouin lines (longitudinal phonons). Since in the case of PMPS the internal relaxations, i.e., the 
phenyl flip and methyl group rotation [15] are stronger than in PEMS (no phenyl group), the effect is stronger in PMPS than in PEMS.

Another interesting feature is the difference in temperature dependence of $\Gamma_{B}\left(T-T_{g}\right)$ between PMPS and PEMS. For liquids with a single relaxation process, the classical acoustic relaxation theory predicts a maximum of the sound attenuation coefficient $2 \pi \Gamma_{\mathrm{B}} / \omega_{\mathrm{B}}$ when $\omega_{\mathrm{B}} \tau_{\mathrm{s}}=1$. Since in supercooled liquids the structural relaxation times $\tau_{\mathrm{s}}$ strongly depend on temperature, a maximum should occur in the temperature dependence of $\Gamma_{B}\left(T-T_{g}\right)$ when this condition is fulfilled. In our case the situation is more complex since at least two processes: internal and structural relaxations, contribute to the damping of longitudinal phonons. As we will show later in this work, the temperature dependence of the contribution of internal relaxation to the Brillouin line width is weak and does not exhibit any maximum. Thus, the maximum in $\Gamma_{\mathrm{B}}\left(\mathrm{T}-\mathrm{T}_{\mathrm{g}}\right)$ must be related to the temperature dependence of the structural relaxation. In PEMS the structural relaxation consists of one $\alpha$-process, while in PMPS two processes: $\alpha$ and $\alpha$ ' are observed [6]. It was found that the $\alpha^{\prime}$-process is slower than the $\alpha$-relaxation and the separation in time between these processes in polysiloxanes containing the phenyl group is increasing with molecular weight of the polymer [25]. For PMPS of molecular weight used in this study this separation amounts to three orders of magnitude. The temperature dependence of the relaxation times of the $\alpha$ - and $\alpha^{\prime}$-processes is very similar and can be described by the Vogle-Fulcher-Tammann law [6,25]. Thus, in a temperature scan, the condition for the maximum of the acoustic attenuation coefficient and the Brillouin line width will be met first by the faster $\alpha$-process (at a lower temperature) and then by the slower $\alpha^{\prime}$-process 
(at a higher temperature). This will result in a broadening of the $\Gamma_{\mathrm{B}}\left(\mathrm{T}-\mathrm{T}_{\mathrm{g}}\right)$ maximum, as observed in the case of PMPS.

\section{IV.2.2. Pressure dependence of the longitudinal phonon damping in PMPS}

A way to separate the effect of internal and structural relaxations on the broadening (damping) of the VV Brillouin lines (longitudinal phonons) is to study the effect of pressure on the Brillouin spectra, since the structural relaxation strongly depends on pressure, while the internal relaxations are practically pressure independent, in the pressure range used. We have shown already that such a separation was possible in the case of low molecular weight supercooled liquids $[4,5]$.

From the pressure dependent Brillouin studies of PMPS at three temperatures, the temperature dependence of the contributions of the internal $\Gamma_{0}$ and structural $A$ relaxations to the width of the VV Brillouin line (damping of the transverse phonons) was obtained, Fig.6. The amplitude A of the contribution of structural relaxation decreases with decreasing temperature and tends to zero at the glass transition temperature $\left(-37^{\circ} \mathrm{C}\right)$. The contribution of the internal relaxation increases with increasing temperature and at high temperature might reach the approximate value of $0.25 \mathrm{GHz}$ in agreement with the residual line width at high temperature obtained in the previous studies [16]. The damping of the longitudinal phonons due to internal relaxation is very efficient in the entire temperature and pressure range studied, due to the fact that the relaxation frequency of the internal processes, especially of the phenyl flip, is very close to that of the longitudinal phonon [15]. 


\section{IV.2.3. Pressure dependence of the transverse phonon damping in PMPS}

In the case of weak coupling of internal relaxations to the translational degrees of freedom, the theory [3] predicts no effect of internal relaxations on the broadening (damping) of the depolarized Brillouin lines (transverse phonons). On the other hand, we have observed such an effect in the case of low molecular weight supercooled liquids [4,5]. Thus, it is interesting to check if a similar disagreement between theoretical predictions and experiment occurs for polymeric supercooled liquids.

From the temperature and pressure dependence of the depolarized Brillouin spectra measured for PMPS it was possible to separate the contributions to the Brillouin width related to the internal and structural relaxations, Fig.10. Obviously, Fig.10, the internal relaxation contributes substantially to the width (damping) of the $\mathrm{VH}$ Brillouin spectra (transverse phonons), in contradiction to the theoretical predictions [3]. The internal contribution to the VH Brillouin spectra is similar to that of VV spectra and increases slightly, being the dominating one at low temperatures close to $\mathrm{T}_{\mathrm{g}}$. The amplitude of the contribution due to the structural relaxation decreases to zero at $\mathrm{T}_{\mathrm{g}}$, as expected.

\section{IV.2.4. Comparison of phonon damping in low molecular weight and polymeric supercooled liquids}

The comparison of the half-width $\Gamma_{\mathrm{B}}$ of the polarized (VV) Brillouin lines (related to the damping of the longitudinal phonons), measured at different temperatures above the glass transition temperature $\mathrm{T}_{\mathrm{g}}$ for low molecular weight van der Waals supercooled liquids 
ortho-terphenyl (OTP), 1,1'-bis(p-methoxy-phenyl) cyclohexane (BMPC) and 1,1'-di(4methoxy-5-methylphenyl) cyclohexane (BMMPC) and polymeric (PMPS, PEMS) supercooled liquids, is shown in Fig 11.

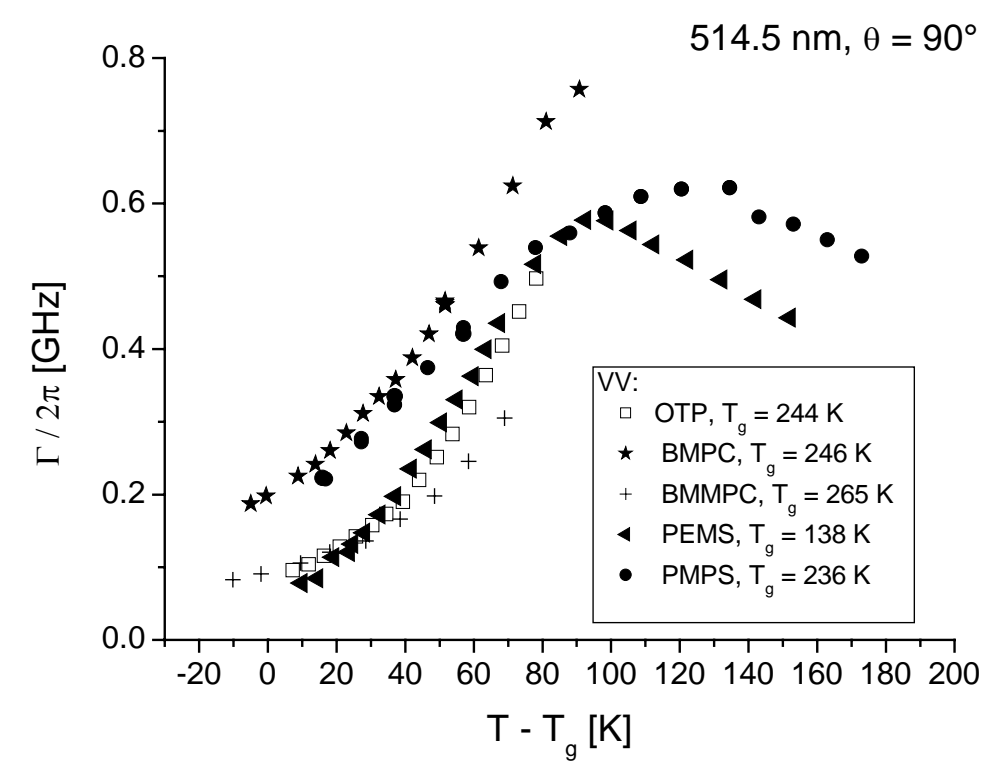

Fig. 11. Temperature dependence of the half-width of the polarized Brillouin lines measured in OTP, BMPC and BMMPC, taken from [5] and in PEMS and PMPS.

It is clearly seen that $\Gamma_{\mathrm{B}}\left(\mathrm{T}_{\mathrm{g}}\right)$ is substantially different from zero for all of these liquids. Additionally, for liquids with strong internal relaxations (BMPC, PMPS) the value of $\Gamma_{\mathrm{B}}\left(\mathrm{T}_{\mathrm{g}}\right)$ is much larger than for those with weak internal relaxation processes (BMMPC, OTP, PEMS). This is in agreement with predictions of the theoretical model [3].

In Fig. 12, the comparison of the half-width $\Gamma_{\mathrm{BT}}$ of the depolarized Brillouin lines (related to the damping of transverse phonons) measured for OTP, BMPC, BMMPC and PMPS above $\mathrm{T}_{\mathrm{g}}$ is shown. 


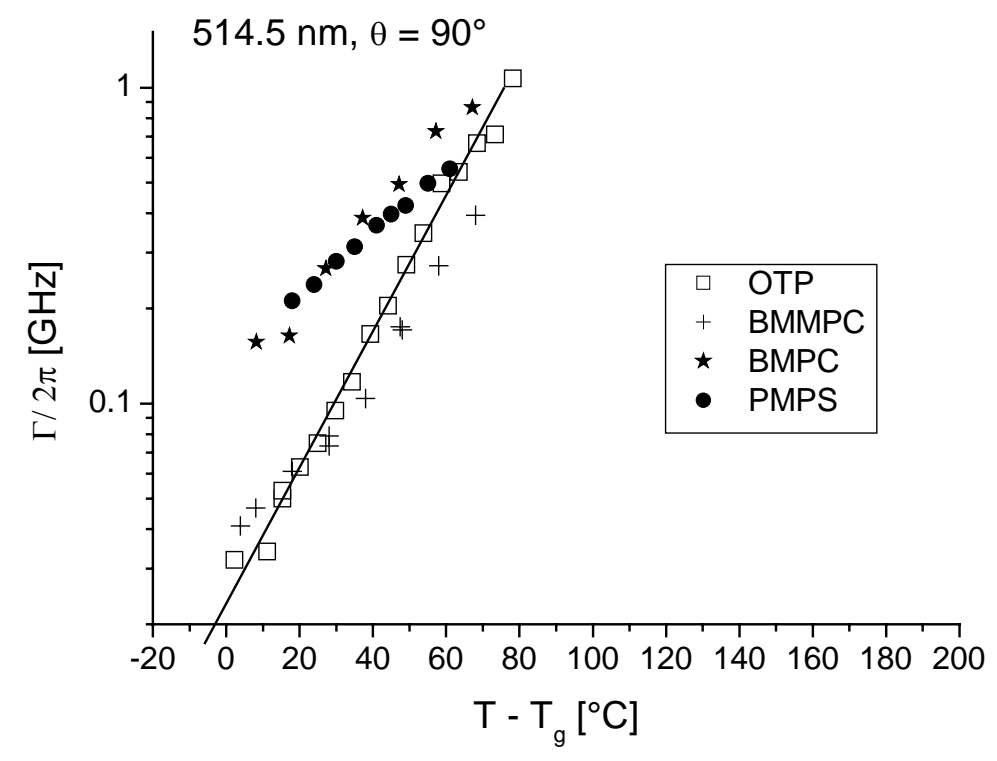

Fig. 12. Temperature dependence of the half-width of the depolarized Brillouin lines measured in OTP, BMPC and BMMPC, taken from [4] and in PEMS and PMPS.

In the case of OTP and BMMPC, i.e. liquids with weak internal relaxations, the $\Gamma_{\mathrm{BT}}\left(\mathrm{T}_{\mathrm{g}}\right)$ values approach zero, i.e. are comparable to the resolution function of the Tandem-FPI used. On the contrary, in the case of liquids having strong internal relaxations (BMPC, PMPS) the values of $\Gamma_{\mathrm{BT}}\left(T_{\mathrm{g}}\right)$ are still quite high.

Thus, liquids having weak internal relaxations (OTP, BMMPC, PEMS) behave according to the predictions of the theoretical model [3], i.e. they exhibit additional broadening (damping) of the VV Brillouin lines (longitudinal phonons) and no such effect in VH Brillouin spectra (transverse phonons). On the other hand, liquids having strong internal relaxations (BMPC, PMPS) exhibit not only additional broadening (damping) of the VV Brillouin lines (longitudinal phonons), in agreement with the theory, but also additional 
broadening (damping) of the VH Brillouin lines (transverse phonons) in contradiction to the theoretical predictions.

\section{Conclusions}

Polarized and depolarized Brillouin spectra were measured for PMPS as function of temperature and pressure and for PEMS as function of temperature, by means of a Tandem Fabry-Perot interferometer, and the positions and line width of the corresponding Brillouin lines were determined.

In the temperature dependent studies on both polymers at ambient pressure, PMPS - a polymer with stronger internal relaxations (phenyl and methyl group rotations) exhibited much larger residual broadening at $\mathrm{T}_{\mathrm{g}}$ than PEMS - a polymer with weaker internal processes (ethyl and methyl group rotations). This residual broadening is due to the contribution of the intra-molecular processes, since at $\mathrm{T}_{\mathrm{g}}$, as we have shown, the amplitude of the structural relaxation contribution is negligible. In this respect the PMPS and PEMS polymers behave in a similar way to two groups of low molecular weight liquids with strong (BMPC) and weak (BMMPC, OTP) internal relaxations, respectively $[4,5]$.

It is also shown that the pressure dependent measurements allow for the separation of the contributions due to intra-molecular and structural relaxations to the polarized and depolarized Brillouin line widths and the damping of longitudinal and transverse phonons, respectively.

From the pressure dependent polarized Brillouin line width of PMPS the temperature dependent contributions of internal and structural relaxations to the $\Gamma_{\mathrm{B}}$ and the damping 
of longitudinal phonons were estimated. The internal contribution is changing from 0.12 to $0.18 \mathrm{GHz}$ and the structural one from 0 to $0.25 \mathrm{GHz}$ in the temperature range from $\mathrm{T}_{\mathrm{g}}$ to $\mathrm{T}_{\mathrm{g}}+60 \mathrm{~K}$.

From the pressure dependent depolarized Brillouin line width of PMPS the temperature dependent contributions of internal and structural relaxations to the $\Gamma_{\mathrm{BT}}$ and the damping of transverse phonons were estimated. The internal contribution is changing from 0.12 to $0.15 \mathrm{GHz}$ and the structural one from 0 to $0.42 \mathrm{GHz}$ in the temperature range from $\mathrm{T}_{\mathrm{g}}$ to $\mathrm{T}_{\mathrm{g}}+60 \mathrm{~K}$.

Thus, we found that in polymers with strong internal relaxations, these processes contribute substantially to both VV (longitudinal) and VH (transverse) Brillouin line width. This is a similar behavior to that observed earlier in a low molecular weight liquid with a strong internal relaxation (BMPC) $[4,5]$.

The experimental data obtained for both low molecular weight and polymeric supercooled liquids having strong intra-molecular relaxations indicate a substantial contribution of these relaxations to the broadening (damping) of the VV Brillouin lines (longitudinal phonons), in agreement with the theory [3] derived in the limit of weak coupling of internal relaxations to the translational degrees of freedom of the molecules. On the other hand, the experimentally measured, for such liquids, substantial contribution of internal relaxations to the broadening (damping) of $\mathrm{VH}$ Brillouin lines (transverse phonons) is in contradiction to the theory, which explicitly predicts no such effect. 


\section{Acknowledgements}

This work was supported by the Polish Ministry of Science and Higher Education (grant No 1 P03B 083 26) and European Network of Excellence "SoftComp" (No. S080118). We thank Th. Wagner from MPI-Polymerforschung, Mainz for the PMPS and PEMS samples. 


\section{References}

[1] A. Aouadi, C. Dreyfus, M. Massor, R. M. Pick, T. Berger, W. Steffen, A. Patkowski, and C. Alba-Simionesco, J. Chem. Phys. 112, 9860-9873 (2000).

[2] C. Dreyfus, A. Aouadi, R.M. Pick, W. Steffen, T. Berger, and A. Patkowski, Europhys. Lett., 42, 55 (1998).

[3] R. Zwanzig, J. Chem. Phys., 43, 714 (1965).

[4] A. Patkowski, J. Gapinski, G. Meier, H. Kriegs, A. Le Grand and C. Dreyfus, Europhys. Lett., 73, 607-613 (2006).

[5] A. Patkowski and J. Gapinski, G. Meier and H. Kriegs, J. Chem. Phys. 126, 014508$1-014508-9(2007)$

[6] H. Kriegs, J. Gapinski, G. Meier, M. Paluch, S. Pawlus and A. Patkowski, J. Chem. Phys. 124, 104901 (2006).

[7] S. Saito, H. Sasabe, T. Nakajima and K. Yada; J. Polym. Sci. Part A2, 6, 1297 (1968)

[8] G. Williams, Trans. Faraday Soc., 62, 2091 (1966)

[9] G. Fytas, G. Meier, A. Patkowski and Th. Dorfmüller, Coll. Polym. Sci, 260, 949 (1982)

[10] B.Y. Li, D.Z. Jiang, G. Fytas, and C.H. Wang, Macromolecules 19, 778-784 (1986)

[11] G. Meier, F. Kremer, G. Fytas, A. Rizos, J. Polym. Sci. Part B: Polym. Phys. 34, $1391-1401(1996)$

[12] G.D. Patterson, Ann. Rev. Mater. Sci. 13, 219-245 (1983)

[13] M. Beiner, Macromol. Rapid Commun. 22, 869-895 (2001)

[14] G. Fytas, Y-H. Lin, and B. Chu, J. Chem. Phys. 74, 3131 (1981) 
[15] G. Meier, F. Fujara, and W. Petry, Macromolecules, 22, 4421 (1989)

[16] C.H. Wang, G. Fytas, and J. Zhang, J. Chem. Phys. 82, 3405 (1985)

[17] D. Boese, B. Momper, G. Meier, F. Kremer, J.U. Hagenah, and E.W. Fischer, Macromolecules 22, 4416 (1989).

[18] M. Paluch, R. Casalini, A. Patkowski, T. Pakula, and C.M. Roland, Phys. Rev. E, 68, 031802 (2003).

[19] G. Monaco, D. Fioretto, L. Comez, and G. Ruocco, Phys. Rev. E, 63, 061502 (2001)

[20] R. Zwanzig, in Lectures in Theoretical Physics, edited by W. Brittin (WileyInterscience, New York, 1961), Vol. 3, pp. 106-141; H. Mori, Prog. Theor. Phys. 33, 423 (1965).

[21] B.J. Berne and R. Pecora, Dynamic Light Scattering (Dover Publications, Inc., Mineola, New York, 2000), p. 243

[22] G. Meier and H. Kriegs, Rev. Sci. Instrum., submitted

[23] C. Dreyfus, A. Aouadi, J. Gapinski, M. Matos-Lopes, W. Steffen, A. Patkowski, and R.M. Pick, Phys. Rev. E 68, 011204 (2003)

[24] EPAPS

[25] A. Patkowski, J. Gapinski, T. Pakula, G. Meier, Polymer, 47, 7231-7240 (2006). 